\title{
Novel method of combined photon beam radiotherapy and brachytherapy for treatment of extensive advanced scalp squamous cell carcinoma
}

\author{
Mostafa Farzin, MD',2, Ramin Jaberi, PhD³, Fatemeh Jafari, MD 2,4 , Mohsen Najafi, MSc ${ }^{4}$, Kasra Kolahdouzan, MD²,4 \\ 'Brain and Spinal Cord Injury Research Center, Neuroscience Institute, Tehran University of Medical Sciences, Tehran, Iran, ${ }^{2}$ Radiation \\ Oncology Research Center, Imam Khomeini Hospital Complex, Tehran University of Medical Sciences, Tehran, Iran, ${ }^{3}$ Radiation Oncology \\ Department, Yas General Hospital, Tehran, Iran, ${ }^{4}$ Department of Radiation Oncology, Cancer Institute, Imam Khomeini Hospital Complex, \\ Tehran University of Medical Sciences, Tehran, Iran
}

\begin{abstract}
Radiotherapy of extensive malignant scalp lesions has always been a challenge for radiation oncologists considering the proximity of critical structures.

We treated a 39-year-old patient with extensive scalp squamous cell carcinoma with application of adjuvant concurrent chemoradiation and external beam radiation therapy (EBRT), followed by high-dose-rate (HDR) surface brachytherapy boost using an exclusively designed mould, and assessed the radiation dose reaching planning target volume, brain, and optic structures.

Comparison between conventional planning with sole EBRT and combined treatment planning with EBRT and brachytherapy, assured the use of the latter treatment to avoid high radiation doses from reaching critical organs at risk without compromising the required dose for planning target volume.

Key words: scalp squamous cell carcinoma, brachytherapy, radiation therapy.

\section{Purpose}

In 2018, non-melanoma skin cancer (NMSC) was the second most prevalent cancer in the world [1], and cutaneous basalcell carcinomas (BCC) and squamous cell carcinomas (SCC) were the most common pathologic sub-groups of this entity. The mainstay for treatment of NMSC is surgery [2]. However, radiation therapy can be applied for definitive treatment in patients who are unfit or reluctant to undergo surgery [3]. Also, radiation is used as an adjuvant treatment to surgery in cases of gross or microscopic residual disease. Radiotherapy is suitable for NMSC under the following conditions: 1 . Treatment of primary tumor, 2. Adjuvant to lesions with close or positive margins after excision, 3. High-risk of recurrence after excision, 4. Recurrent tumors [4].

Numerous benefits of high-dose-rate (HDR) brachytherapy over external beam radiation therapy (EBRT) demonstrate HDR brachytherapy a more favorable method to deliver a higher dose of conformal radiation to the skin, with minimum toxicity to the adjacent tissues and better cosmetic results. Current brachytherapy techniques for skin treatment include electronic brachyther- apy, radionuclide brachytherapy, brachytherapy with moulds or flaps, and interstitial brachytherapy [5]. HDR brachytherapy with customized applicators has shown to be highly efficient for complex scalp lesions [6]. This report shares our experience of treating a patient with an extensive scalp squamous cell carcinoma and bilateral cervical lymphadenopathies, who was treated with adjuvant EBRT followed by HDR brachytherapy boost to the scalp through an exclusively designed mould.

All of the following information is reported with the patient's consent.

\section{Case presentation}

A 39-year-old man presented to the radiation oncology clinic for surgeon's consultation, requiring an adjuvant radiation to his scalp after extensive resection of a primary scalp lesion.

Six years earlier, the patient had developed a scalp lesion on the right parietal side and underwent a resection. The initial pathology report indicated squamous cell carcinoma. After nearly 6 years of being disease-free, he noted a new lesion on the same spot, which had dissem- 

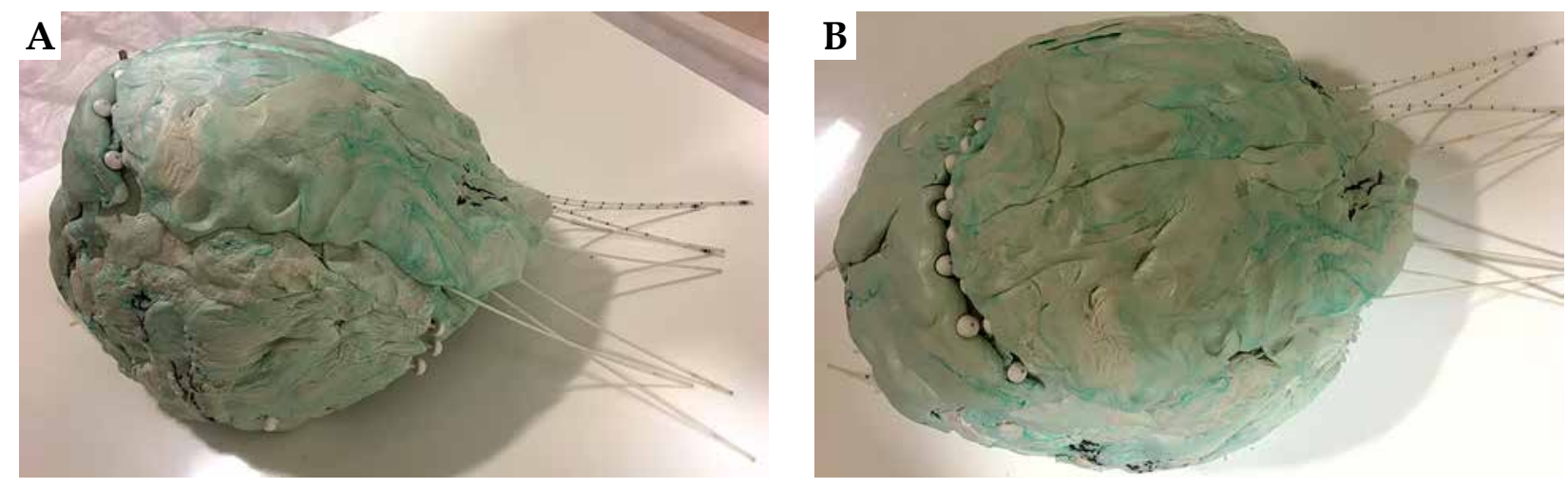

Fig. 1. Exclusive mould created for brachytherapy of extensive scalp squamous cell carcinoma. A) Left lateral view, the anterior portion of the mould is on the left side of the image. B) Vertex view, the anterior portion of the mould is on the left side of the image

inated and involved almost all scalp tissue within one month. On magnetic resonance imaging, a vegetative and enhancing mass over the right parietal bone measuring $74 \mathrm{~mm} \times 74 \mathrm{~mm} \times 38 \mathrm{~mm}$ was noted, with suspicious invasion to the external table of bone and adjacent bone marrow. Multiple suspicious lymphadenopathies on the left cervical levels 2 and 5 as well as the left occipital and parotid regions were reported with short axis diameter (SAD) of about $10 \mathrm{~mm}$. A core needle biopsy of suspicious lymph nodes indicated squamous cell carcinoma and later, the patient underwent an extensive scalp surgery for resection of the lesions. The skin defect was covered with a rotational flap and skin grafting.

The surgical pathology report indicated a 9-centimeter large tumor with $2.5 \mathrm{~cm}$ thickness, and the tumor was classified as poorly differentiated SCC. The tumor showed lymphovascular invasive features, but perineural invasion was not detected. Deep margin of the excised tissue was affected by the tumor; however, bone trabeculae and intervening marrow were not involved. The surgeon additionally resected two other suspicious lesions on the

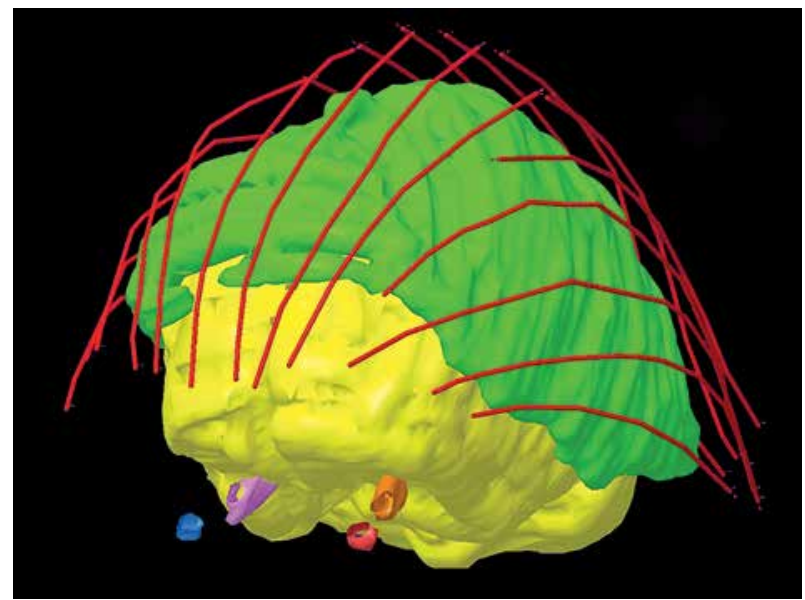

Fig. 2. 3D illustration of brachytherapy catheters' spatial configuration adjacent to planning treatment volume (in green), brain (in yellow), and optical structures (optic nerves in purple and orange, optic lenses in red and blue) by HDR plus treatment planning system right frontal and left parietal regions of the scalp. The former was reported to be actinic keratosis grade 3 (Bowen's disease), while the latter showed features of early invasive SCC in a background of actinic keratosis with a thickness of $2.2 \mathrm{~mm}$ and close medial margin ( $3 \mathrm{~mm}$ ).

We intended to treat the patient with adjuvant radiotherapy to a total dose of $66 \mathrm{~Gy}$ with $6 \mathrm{MV}$ photons. However, it was soon realized that such a procedure would require a significant brain volume to receive high radiation doses. For this reason, planning and treatment was divided into two steps, including EBRT and brachytherapy (BT).

For EBRT treatment, the patient was immobilized using U-shape thermoplastic head mask. A fine cut (1 mm) computed tomography (CT) scan was acquired and transferred to Eclipse 13 planning software (Eclipse, Varian Medical Systems, USA). Clinical target volume (CTV) was defined as the total thickness of skin down to the periosteum of the skull bone. Also, a $10 \mathrm{~mm}$ radial margin to the surgical scar was considered. Planning target volume (PTV) was delineated as CTV with a $5 \mathrm{~mm}$ margin. Using 3D conformal radiotherapy, scalp (including the graft tissue, tumor bed, and surgical scars) and neck was irradiated with $6 \mathrm{MV}$ photons to a cumulative dose of 42 Gy in 21 daily fractions, including two right and left lateral fields. The treatment of neck was continued for nine more fractions to a cumulative dose of $60 \mathrm{~Gy}$ with $6 \mathrm{MV}$ photons by 3D conformal radiation delivered through two antero-posterior and postero-anterior fields bilaterally to cover cervical nodal levels 2 to 5 . The patient received concurrent weekly cisplatin infusion, with a prescribed dose of $35 \mathrm{mg} / \mathrm{m}^{2}$. EBRT treatment was delivered by Elekta Compact linear accelerator.

For BT part of treatment, we prepared an exclusive mould and inserted 21 brachytherapy catheters in a specific pattern (Figures 1 and 2). The mould was created with a waxy material to cover all diseased scalp tissue, and its thickness varied between 9 and $21 \mathrm{~mm}$, depending on the thickness of reconstructed scalp tissue in different loci. Eight confronting pairs of catheters, with a distance of about $1 \mathrm{~cm}$ from each other, were placed through frontoparietal part of the mould towards frontal and occipital directions, and 5 supplemental catheters were placed 

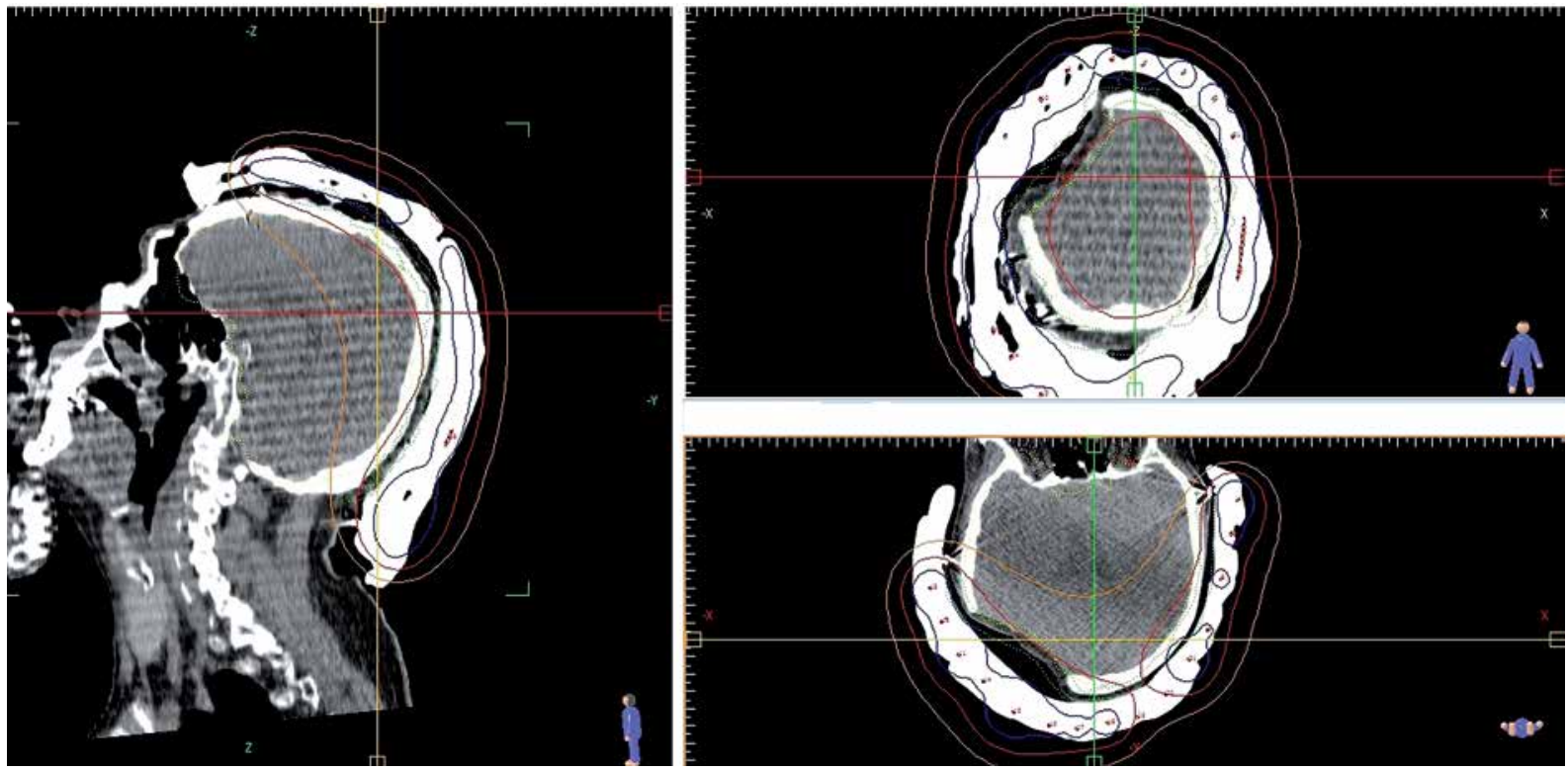

Fig. 3. Dose distribution curves in sagittal, coronal, and axial planes of simulated CT scan of the patient wearing the exclusive mould in prone position. In this image, blue, red, and orange lines demonstrate $\mathrm{V}_{150}, \mathrm{~V}_{100}$, and $\mathrm{V}_{71}$ (percent volume receiving 2 Gy), respectively

through occipitotemporal part of the mould to cover the remaining scalp skin. The catheters had a constant distance from the upper surface of the mould.

Planning CT scan with $1 \mathrm{~mm}$ slice thickness was acquired for patient with mould fixed in place with a U-shape thermoplastic head mask in prone position. Images were transferred to HDR plus planning system, where catheters' position was reconstructed (Figure 3). A TG-43 task group algorithm was used for HDR brachytherapy treatment planning. The patient received high-dose-rate brachytherapy boost with Cobalt-60 MultiSource HDR brachytherapy unit (Eckert \& Ziegler BEBIG GmbH, Germany) to a total dose of 22.4 Gy in 8 once-daily fractions. Each treatment session lasted for nearly thirty-two minutes. Considering an $\alpha / \beta$ ratio of $10 \mathrm{~Gy}$ for tumor, the equieffective dose in $2 \mathrm{~Gy}$ per fraction $\left(\mathrm{EQD}_{2}\right)$ of the brachytherapy boost was calculated to be 23.9 Gy, according to Dale et al. [7]. Therefore, the patient received $66 \mathrm{~Gy}$ to the bed of the primary scalp lesion through combined radiotherapy with brachytherapy boost without surpassing the radiation limit to the brain.

For better comprehension of the advantages of brachytherapy boost treatment, we also created a treatment plan with sole EBRT application to the final dose of $66 \mathrm{~Gy}$ in 33 fractions, with two lateral fields to the whole scalp tissue and bilateral cervical nodal levels, similar to the initial plan. Brain and orbital structures, including optic nerves, chiasma, and lenses were contoured as organs at risk, and dose volume histograms (DVH) were calculated for both plans (Figures 4 and 5, Table 1 [8]).

The patient experienced temporary grade 1 skin erythema and hyperpigmentation during the brachytherapy treatment, which resolved in two weeks after the completion of treatment.

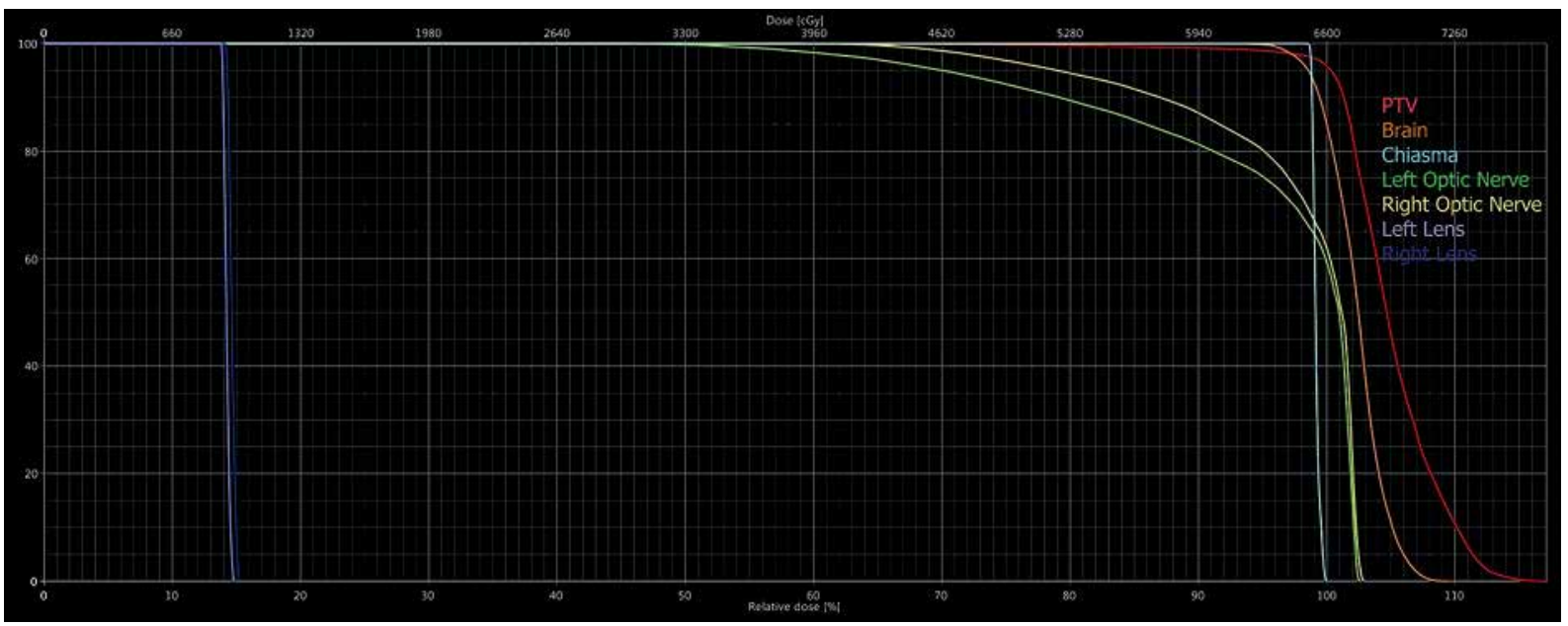

Fig. 4. Dose-volume histogram of planning with external photon beam radiation therapy of 2 Gy per fraction 


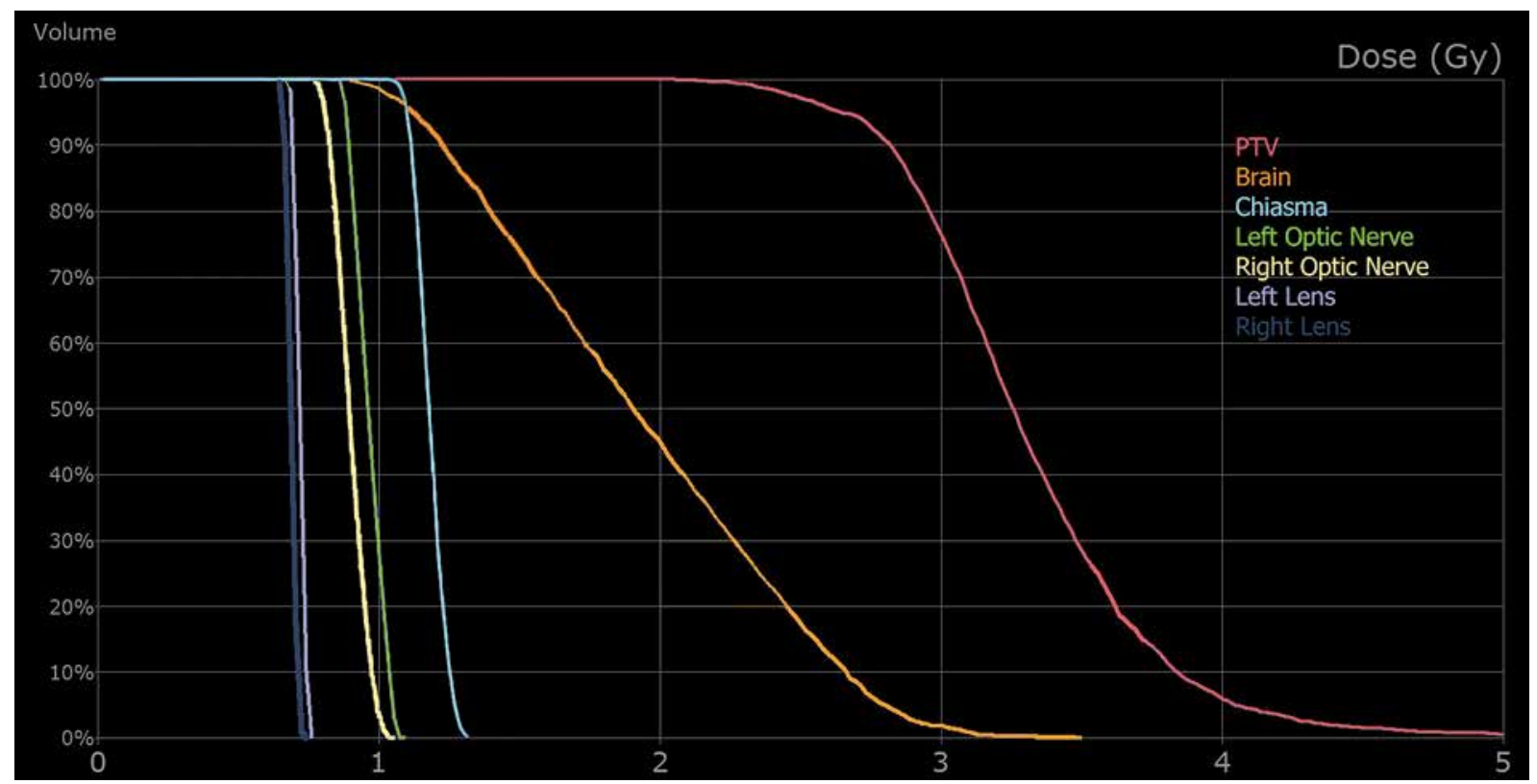

Fig. 5. Dose-volume histogram of planning with high-dose-rate brachytherapy of 2.8 Gy per fraction

Table 1. Comparison of radiobiology doses for planning target volume, brain, and optical structures in two radiation planning techniques by external beam radiation therapy (EBRT) alone and EBRT plus brachytherapy boost

\begin{tabular}{|c|c|c|}
\hline & $\begin{array}{l}\text { EBRT }+ \text { BT } \\
\text { boost }^{*} \\
\text { (scalp: } \\
42 \mathrm{~Gy} / 21 \mathrm{fx}+ \\
22.4 \mathrm{~Gy} / 8 \mathrm{fx})\end{array}$ & $\begin{array}{c}\text { EBRT } \\
(66 \mathrm{~Gy} / \\
33 \mathrm{fx})\end{array}$ \\
\hline \multirow{2}{*}{\multicolumn{3}{|c|}{$\begin{array}{l}\text { PTV } \\
\text { Volume }\left(387.1 \mathrm{~cm}^{3}\right)\end{array}$}} \\
\hline & & \\
\hline$\underline{\mathrm{D}_{90}(\mathrm{~Gy})}$ & 67.1 & 67.9 \\
\hline$\underline{\mathrm{D}_{98}(\mathrm{~Gy})}$ & 61.9 & 65.4 \\
\hline $\mathrm{V}_{50}(\%)$ & 100.0 & 100.0 \\
\hline$V_{100}(\%)$ & 90.8 & 97.7 \\
\hline $\mathrm{V}_{200}(\%)$ & 0 & 0 \\
\hline \multicolumn{3}{|l|}{$\begin{array}{l}\text { Brain } \\
\text { Volume }\left(1,584 \mathrm{~cm}^{3}\right)\end{array}$} \\
\hline $\mathrm{D}_{90}(\mathrm{~Gy})$ & 44.7 & 58.2 \\
\hline $\mathrm{D}_{98}(\mathrm{~Gy})$ & 37.6 & 48.3 \\
\hline$\underline{V_{50}(\%)}$ & 79.5 & 99.8 \\
\hline $\mathrm{V}_{100}(\%)$ & 5.0 & 67.9 \\
\hline$V_{200}(\%)$ & 0 & 0 \\
\hline \multicolumn{3}{|c|}{ Optical structures (maximum dose) } \\
\hline Right optic nerve (Gy) & 49.0 & 68.7 \\
\hline Left optic nerve (Gy) & 49.7 & 68.5 \\
\hline Optic chiasma (Gy) & 50.5 & 66.8 \\
\hline Right lens (Gy) & 10.0 & 10.8 \\
\hline Left lens (Gy) & 10.5 & 10.5 \\
\hline \multicolumn{3}{|c|}{$\begin{array}{l}\text { *The total dose in } E Q D_{2} \text { for } E B R T+B T \text { plan was } 65.9 \text { Gy. All treatment doses } \\
\text { in the table are reported as } E Q D_{2} \text {. The predefined } \alpha / \beta \text { ratio for tumor, brain, } \\
\text { optic nerve, chiasma, and lens were } 10,2.1,3,3, \text { and } 1.2 \text {, respectively [8]. PTV } \\
\text { - planning target volume, Di-the dose required to cover i percent of volume, } \\
\text { Vi - percent of volume receiving i percent of the dose, EBRT - external beam } \\
\text { radiation therapy, BT - brachytherapy, fx-fractions }\end{array}$} \\
\hline
\end{tabular}

Four months after brachytherapy to the scalp lesion, the patient remains disease-free without an evidence of recurrence, with acceptable cosmetic results considering the serious and extensive surgery (Figure 6). Fortunately, no latent skin toxicity was evident, and he did not complain of any treatment-related side effects. Neurological and physical examination were typical.

\section{Discussion}

Skin surface brachytherapy with customized mould is an appropriate treatment for irregular and large skin lesions due to an adequate mould-catheter geometry that can be achieved [9].

The American Brachytherapy Society (ABS) guidelines [10] for skin surface brachytherapy published in 2001 stated that mould HDR brachytherapy is considered suitable for lesions of less than $5 \mathrm{~mm}$ thick $[3,4,7]$. However, in our opinion, the evidence behind this $5 \mathrm{~mm}$ cut-off is questionable [11]. In the presented case, the patient had a $2.5 \mathrm{~cm}$ thick lesion before surgery and although extensive surgery was performed with a deep margin involvement, our contoured PTV had a depth of more than $5 \mathrm{~mm}$.

When considering sole treatment with mould surface brachytherapy for such an extensive lesion, one should also bear in mind the inherent flaws, such as mould-skin air gaps and skin surface dose inconsistencies, when PTV has a depth of more than $5 \mathrm{~mm}$. Moreover, due to the need for neck radiotherapy, this method was unfavorable.

The biopsy-proven bilateral cervical lymphadenopathies and suspicious bone involvement in pre-op MRI (although, based on surgical pathology report, the latter was ruled out) required a treatment with EBRT at least to cover the involved cervical lymph nodes (levels 2-5). However, due to the extensive lesion of the scalp, exceeding the dose limits for critical structures (such as brain parenchyma, brain stem, chiasma, optic nerves, hippocampi, etc.) would result in excessive neurological deficits in 


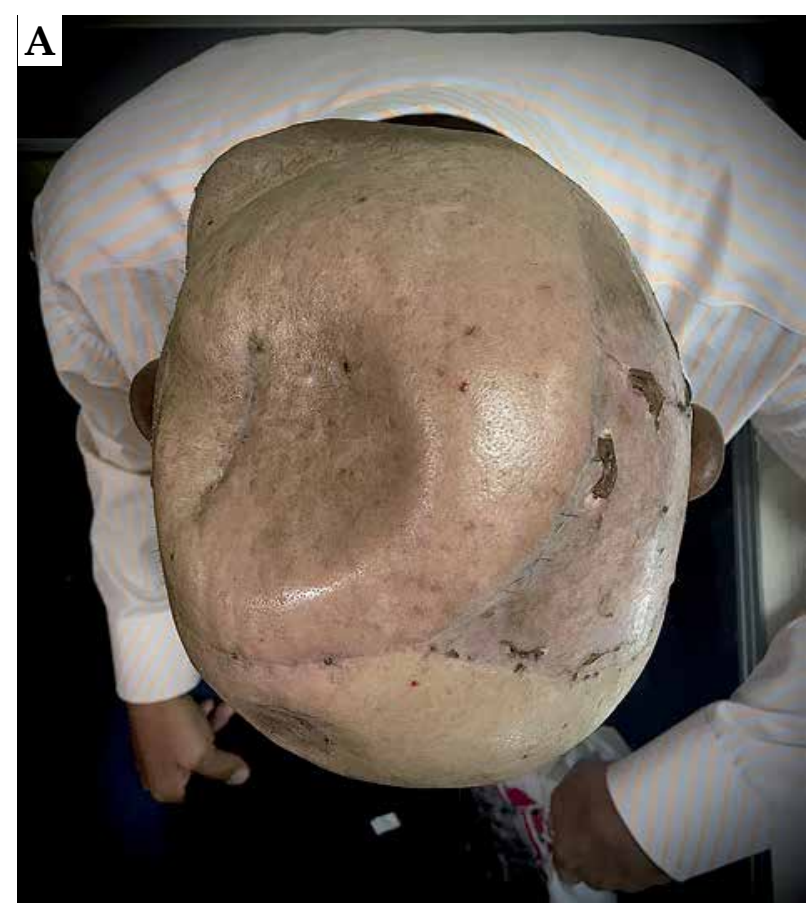

Fig. 6. Clinical follow-up image of the patient at 4 months after the treatment from A) vertex, B) left postero-lateral, and C) right supero-lateral position (all images were photographed with the patient's consent. Background is darkened using Photoshop CS6 software to omit unnecessary details)

the patient. For this reason, a treatment with sole EBRT was not favorable either, especially when considering the young age of the patient.

Therefore, we decided to use EBRT followed by brachytherapy boost to the scalp to cover both the neck and surgical bed, without surpassing the dose constraints for organs at risk.

The novelty of this case remains in the fact that despite an extensive tumor bed requiring radiation, brachytherapy was used with an exclusively designed mould to cover the required CTV. This technique reduced the unnecessary radiation dose to the underlying brain tissue, thus, significantly lowering the subsequent neurologic toxicities $\left(\mathrm{V}_{100}\right.$ for brain $5 \%$ with BT boost vs. $67.9 \%$ without BT boost).

The HDR brachytherapy boost technique has several advantages. Firstly, all concerns about organ at risk (OAR) dose constraints are readily resolved. Also, simple treatment delivery and setup reproducibility reduce the inter-fraction positional errors to minimum. With brachytherapy, the treatment is much more tolerable for the patient with the least radiation-related toxicities, and a higher dose can be safely delivered to the PTV. A previous study by Wojcicka et al. [12] in 2009 reported that HDR brachytherapy plans for extensive scalp lesions were more conformal than IMRT or photon-electron treatment techniques. Contrary to the moulding technique used by Wojcicka and colleagues, the exclusive mould that was created for our patient had a heterogenous thickness ac-
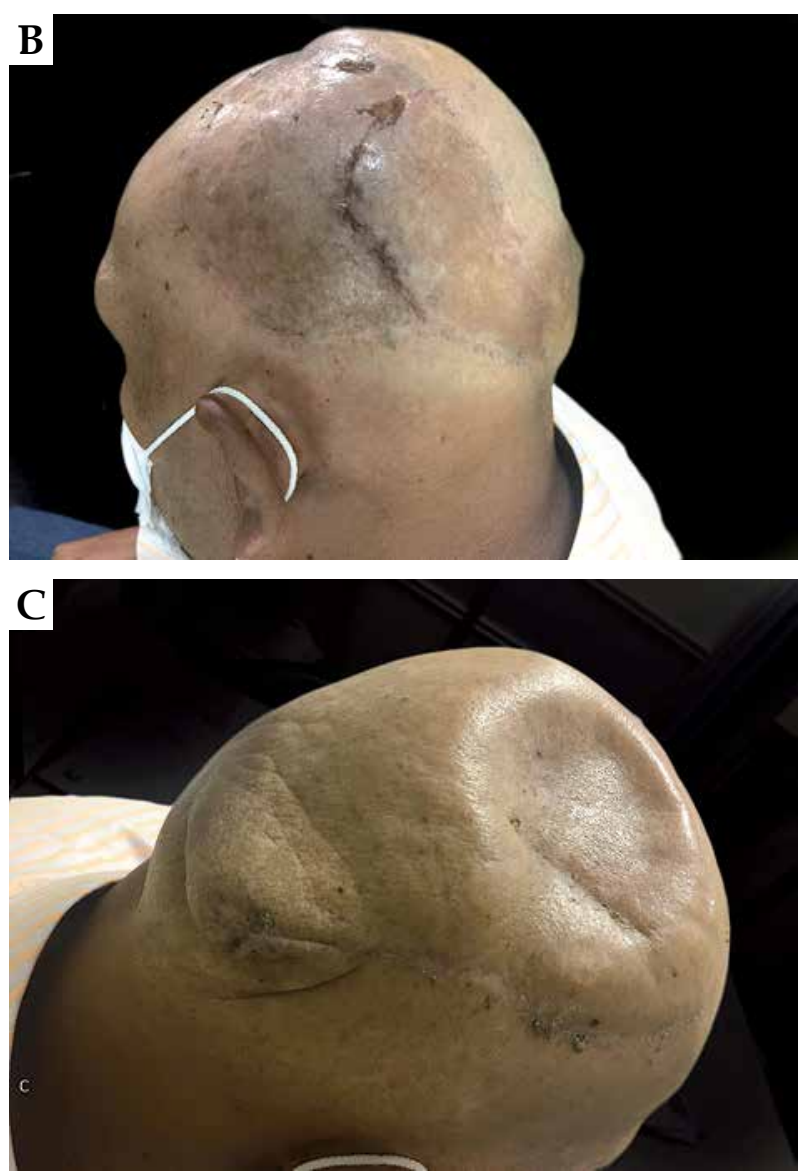

cording to the skin surface irregularities. The mould was thicker over the craniotomy defect area. This different placement of catheters on the skin surface, allowed for delivery of the required dose to deeper parts of the scalp.

Disadvantages of treatment with EBRT compared to BT include: 1. Significant dose fall-off in the skin, which mandates the use of bolus material, 2. Dose reduction in the perimeter of round structures, 3. Deeper dose penetration to the underlying tissues beneath the tumor, which results in higher toxicity [13]

Since the last brachytherapy treatment session 4 months ago, the patient was followed up and currently, there is no evidence of the disease. We will continue the follow-up for any signs of recurrence, but so far, cosmetic results and minimal radiation-induced toxicities are satisfying.

\section{Conclusions}

We recommend that in patients with an extensive scalp malignant lesion who need adjuvant radiation therapy, mould brachytherapy techniques should be more comprehensively studied, so that subsequent neurologic toxicities can be reduced to minimum.

\section{Acknowledgements}

We would like to thank Naser Zare for his help in providing required data for this article. 


\section{Disclosure}

The authors report no conflict of interest.

\section{References}

1. Non-melanoma skin cancer. Globocan 2020. Available from: https://gco.iarc.fr/today/data/factsheets/cancers/17Non-melanoma-skin-cancer-fact-sheet.pdf.

2. Tanese K, Nakamura Y, Hirai I, Funakoshi T. Updates on the systemic treatment of advanced non-melanoma skin cancer. Front Med (Lausanne) 2019; 6: 160.

3. Rong Y, Zuo L, Shang L, Bazan JG. Radiotherapy treatment for nonmelanoma skin cancer. Expert Rev Anticancer Ther 2015; 15: 765-776.

4. Ouhib Z, Kasper M, Perez Calatayud J et al. Aspects of dosimetry and clinical practice of skin brachytherapy: The American Brachytherapy Society working group report. Brachytherapy 2015; 14: 840-858.

5. Shah C, Ouhib Z, Kamrava Met al. The American Brachytherapy society consensus statement for skin brachytherapy. Brachytherapy 2020; 19: 415-426.

6. Jumeau R, Renard-Oldrini S, Courrech F et al. High dose rate brachytherapy with customized applicators for malignant facial skin lesions. Cancer Radiother 2016; 20: 341-346.

7. Dale RG. The application of the linear-quadratic dose-effect equation to fractionated and protracted radiotherapy. $\mathrm{Br}$ J Radiol 1985; 58: 515-528.

8. Kehwar TS. Analytical approach to estimate normal tissue complication probability using best fit of normal tissue tolerance doses into the NTCP equation of the linear quadratic model. J Cancer Res Ther 2005; 1: 168-179.

9. Rodriguez S, Arenas M, Gutierrez C et al. Recommendations of the Spanish brachytherapy group (GEB) of Spanish Society of Radiation Oncology (SEOR) and the Spanish Society of Medical Physics (SEFM) for high-dose rate (HDR) non melanoma skin cancer brachytherapy. Clin Transl Oncol 2018; 20: 431-442.

10. Nag S, Cano ER, Demanes DJ et al. The American Brachytherapy Society recommendations for high-dose-rate brachytherapy for head-and-neck carcinoma. Int J Radiat Oncol Biol Phys 2001; 50: 1190-1198.

11. Jolly DE, Nag S. Technique for construction of dental molds for high-dose-rate remote brachytherapy. Spec Care Dentist 1992; 12: 219-224.

12. Wojcicka JB, Lasher DE, McAfee SS et al. Dosimetric comparison of three different treatment techniques in extensive scalp lesion irradiation. Radiother Oncol 2009; 91: 255-260.

13. Skowronek J. Brachytherapy in the treatment of skin cancer: an overview. Postepy Dermatol Alergol 2015; 32: 362-367. 